\title{
A Study on the Acoustic Variation to Environment Changes in Virtual and Complex Reality
}

\author{
SeongGeon Bae \\ ${ }^{1}$ School of Software Application, Kangnam University, Gyunggido, Korea.
}

${ }^{I}$ Orcid Id: 0000-0003-3252-0062

\begin{abstract}
This study proposes two methods to enhance acoustic immersion in virtual space and to enhance separation in complex reality. In order to solve the phenomena of the immersion degree that appear because the visual and auditory work in the virtual space, which is the most common problem, the strength and weakness of the sound effect during the screen switching are processed. In order to make use of the sound effects used in the virtual space in the mixed reality and the non-adaptation when reflected in the real environment, the feedback method is presented. This study can be applied to reflect the acoustic elements connecting the virtual space and the real environment in the future and to obtain the highest immersion and the lowest separation. Using this method, it is more effective to reflect the mis-adaptation that occurs when moving from the existing HMD virtual space to another virtual space and moving to the virtual space and the real environment.
\end{abstract}

Keywords: HMD, complex reality, virtual space, sound effect

\section{INTRODUCTION}

In virtual space, acoustic characteristics are very important for user's immersion. This immersion uses HMD for the visual side and the general speaker for hearing. This approach is immersive because the visual aspect is important, but the problem is caused by the use of separate spaces in the auditory segment. These problems have been studied in various places in recent years. So far, there is no sense of space or proper processing of sound effects, so simple sound changes or inappropriate effects are added. Due to this, the sound effect felt by the user was used as a feeling separated from the visual

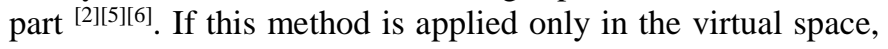
the visual immersion and separation that the user feels can be separated, and in the mixed reality, more and more inconvenience can be felt. These days, the existing method requires a problem or adaptation time because it is diversified from virtual reality to real environment. Therefore, this study proposes a method to solve the adaptation problem to the virtual space and the real environment in various ways. First of all, the problem in the virtual space is that the visual and auditory senses are separated, so it takes time to adapt. In the reality of complex reality, the effects of the virtual space and the ambient sound according to the real environment are not mixed. Recently, dizziness occurs due to problems of immersion in the virtual space and adaptation to the change of the image, especially when the HMD is taken off. These symptoms have been changed to increase the frame or increase the resolution to maximize the visual effect. In addition, resolution is being studied as an important part for applying it to complex reality. As for acoustic problems, it is important to inform in advance of the effect of perceiving sudden screen changes or risk factors that most affect the immersion of the virtual space. However, this method does not greatly affect this in complex environments [1][2][3][4][5][6]. Therefore, in this study, we consider the characteristics of immersion in the virtual space and the effects that can occur in the complex environment by using the feedback method. For example, the separate feeling in the virtual space that affects the degree of immersion is processed through the brightness or correlation of the sudden change in the calculation of the movement of the space or the transition of the screen. And secondly, it proposes to solve the unrealistic sound effects that can appear in the complex reality by adding the acoustical elements of the real environment as feedback. Chapter 2 presents the researched methods to improve the immersion in conventional methods and the limitations in complex reality. In Chapter 3, we propose the proposed method, which is to improve the immersion of sound effects in the virtual space and the feedback using the sense of separation in the composite reality. Chapter 4 presents the results and conclusions of these methods

\section{ACOUSTIC EFFECTS OF VIRTUAL SPACE AND COMPLEX REALITY}

In order to enhance the acoustic immersion in the virtual space, a special device such as the HMD used by the vision is not necessary, but it must reflect all the characteristics of the space and the strength felt in the urban environment. In the usual way, the user adds sound effects around the equipment. It does not affect where the acoustic background is not needed, but there is a problem in immersion in the part that feels thrilling or the part that gives tension. This is because the existing method uses a simple effect because it uses two speakers on both sides of the user. In order to solve these problems have been tried in various ways $[1][2][3][5][7][8][9][10][11][12]$. The usual two-speaker approach is a monotonous, generalized effect that can interfere with 
boredom and immersion. Because it cannot adapt to the change of space in various ways, it is widely used only when changing scenes. As shown in Figure 1, a user wears an HMD and experiences a virtual space and uses sound effects only in the screen transition with the sound effect. When the user moves in this space, the effect is reversed. This mis-adaptation is being tried to solve by considering various methods. In addition, it is difficult to apply to the spatial change or sudden change of the virtual space. The second problem is that in the complex environment of returning from the virtual space to the real environment, the acoustic effect is even less used. This is because the sound effect of the real environment cannot use the sound effect used in the virtual space. This problem has not been solved yet. This is because acoustic effects that can be adapted to environmental changes are needed.

\section{PROPOSED METHOD}

As shown in Figure 2, a histogram is applied to measure the change in brightness of the input image. In order to measure the sudden change, the change of each frame is examined to

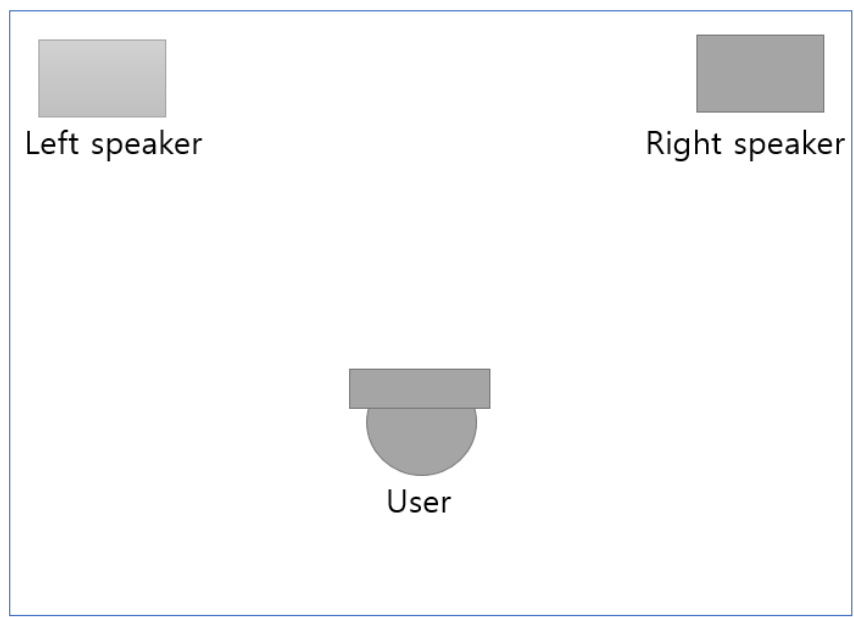

Figure 1. A experience room of a typical virtual space

detect a large change rate. The detected frame applies the sound effect to the condition of the brightness change. The sound effect used 5 patterns in total. The effects of no change, bigger and smaller, smaller and larger, smaller and larger, and smaller and larger.

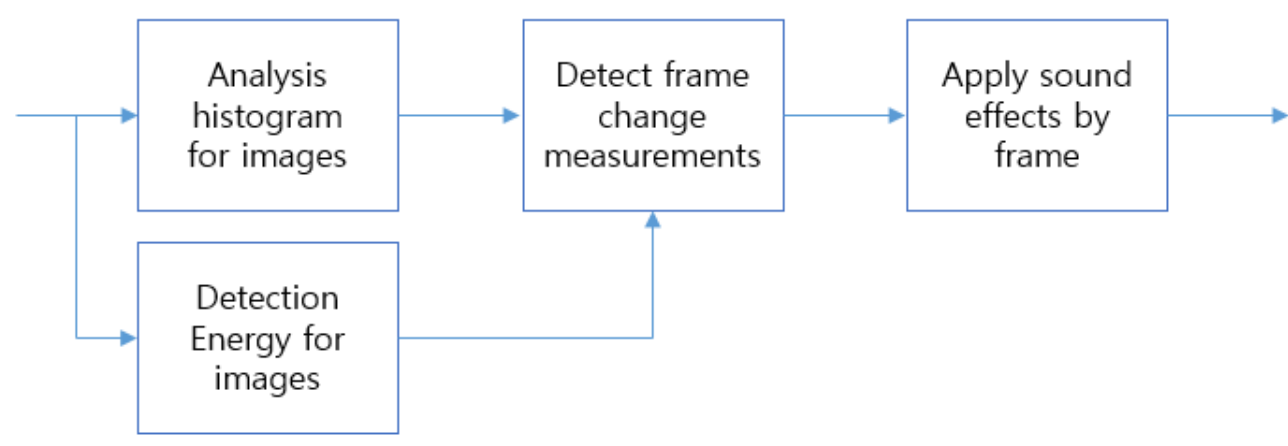

Figure 2. Frame-specific sound effects based on histogram differences
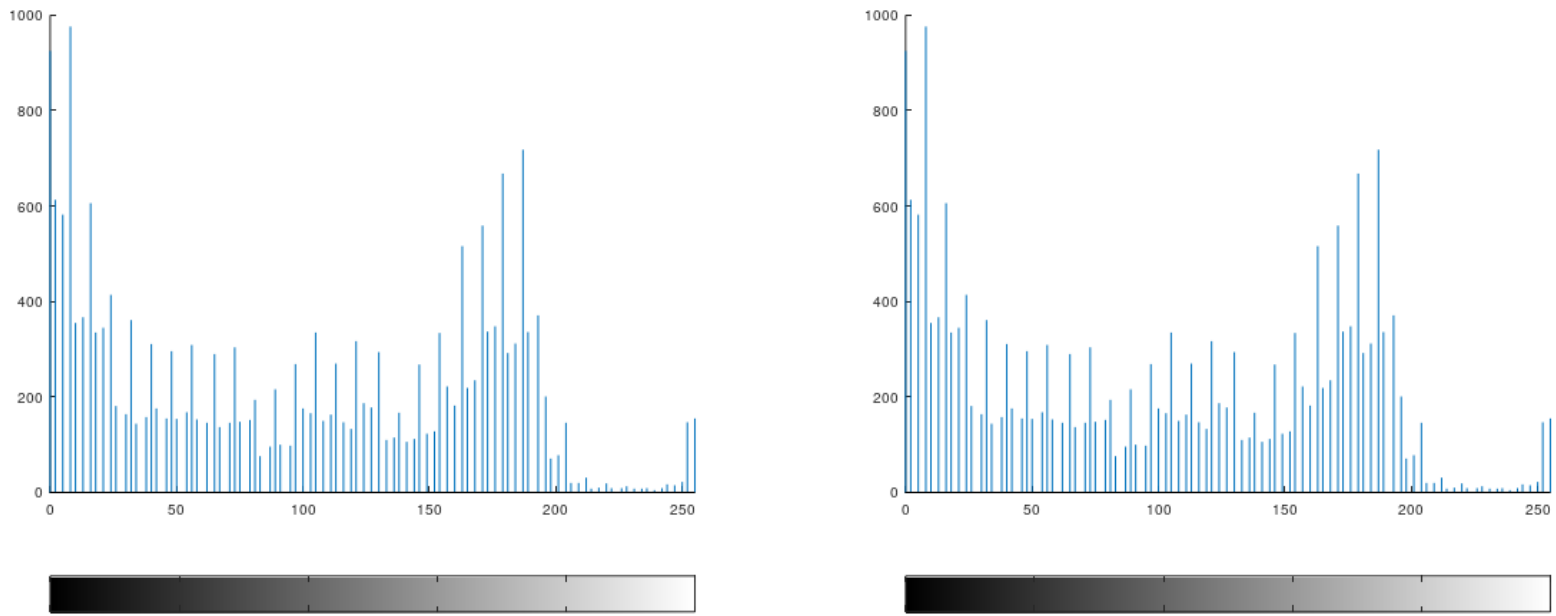

Figure 3. Histogram of a transition of similar screens 
Figure 3 compares the histogram. In general, the histogram shows the same pattern when there is no change. This pattern is treated as an unchanged sound effect. As shown in the figure, it can be seen that the histograms of the frames are
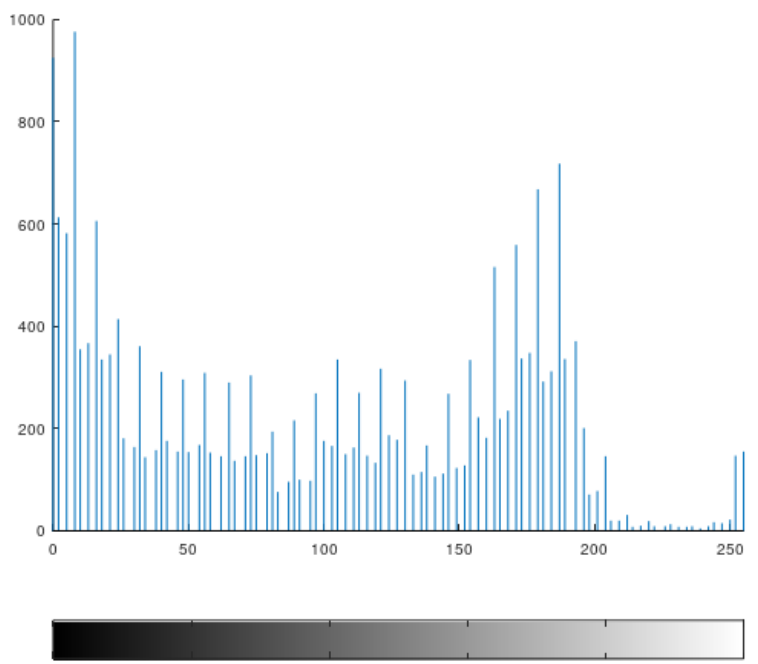

almost similar. Therefore, this study regards these characteristics as unchanged and applies the same to the change degree by obtaining the energy of each frame.

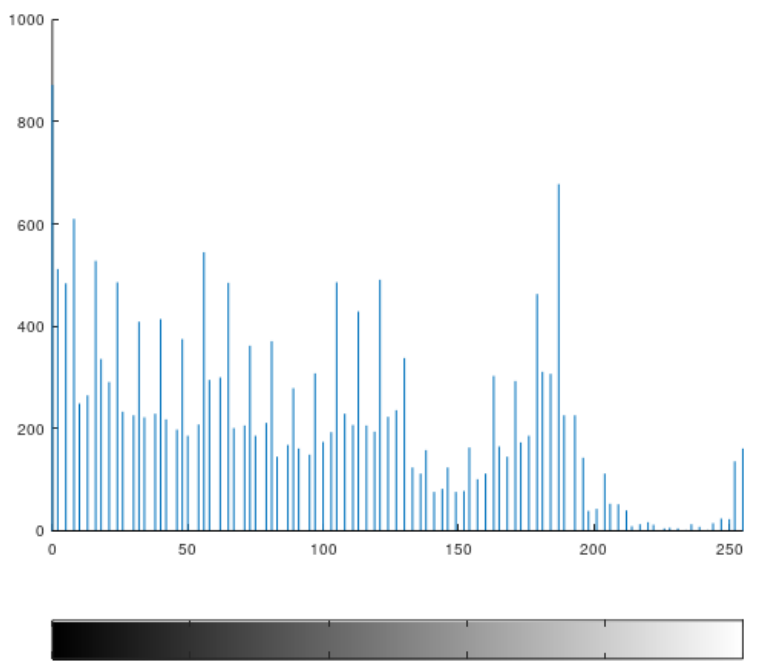

Figure 4. Histogram of screen transitions without similarities

Figure 4 shows the histogram that the change in brightness suddenly changes and the screen changes. In this section, the energy change is also very unstable, and the histogram change is very severe. In order to adapt to various screen changes, strength and weakness are applied to sound effects in four patterns. The same energy comparison was applied to avoid errors in the histogram change.

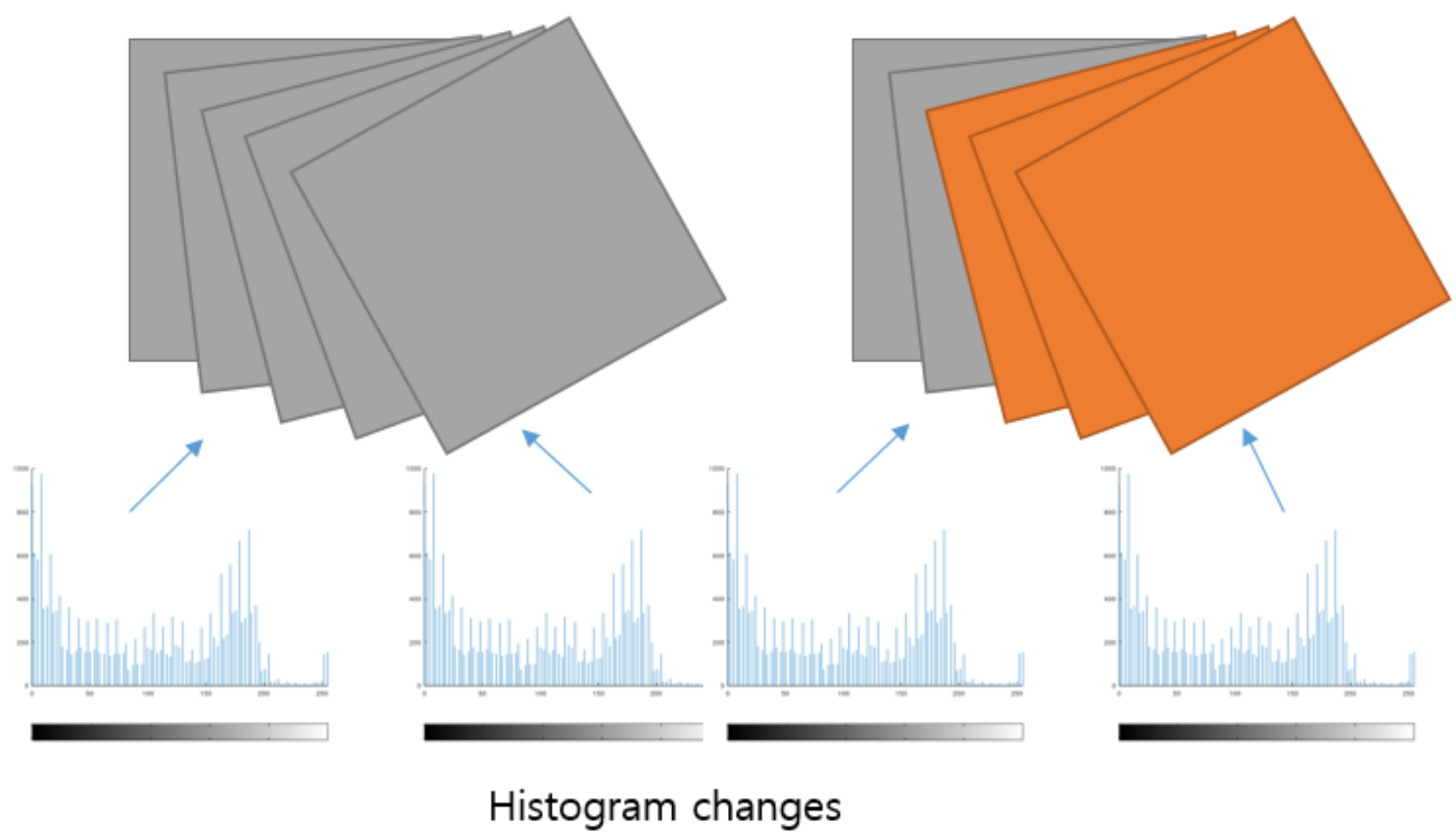

Figure 5. Histogram changes according to screen 
Figure 5 compares a typical frame with a change in the frame where a sudden change occurs. Since the purpose of this study is to investigate the change in the histogram, various attempts have been made. Five sound effect patterns were applied to the patterns examined here.

Table 1. MOS test for Proposed Methods

\begin{tabular}{|c|c|c|c|c|c|c|}
\hline & A & B & C & D & E & Avg. \\
\hline Original Method & 3 & 4 & 4 & 3 & 3 & 3.4 \\
\hline Method 1 & 4 & 5 & 5 & 5 & 4 & 4.6 \\
\hline Method 2 & 5 & 5 & 4 & 5 & 5 & 4.8 \\
\hline
\end{tabular}

\section{CONCLUSION}

This study studied the acoustic effects of immersion in virtual space and separation of complex reality. The general sound effect used in the first virtual space was applied to the sound pressure by measuring the brightness during the screen change and sudden change. Secondly, since the sounds used in the real environment in the mixed reality are very different, the feedbacks of the virtual space and the real environment are additionally used. These two uses are very useful because they reduce the time to apply to the real environment in virtual space and can be applied to various environmental changes. The development of complex reality in the future is to remove the boundary between virtual space and real environment. In order to minimize this problem later, the process of convergence and adaptation to visual changes should be used. In this study, the acoustic effect is used using this method, so it is possible to adapt variously to the visual change. In the future, the method of matching the sound effects according to the spatial change will be studied to be more detailed and realistic.

\section{ACKNOWLEDGEMENT}

This Research was supported by Kangnam University Research Grants (2017).

\section{REFERENCES}

[1] S.G. Bae, M.S. Kim, and M.J. Bae, "On Enhancement Signal Using Non-uniform Sampling in Clipped Signals for LTE Smart Phones," 2013, IEEE ICCE-berlin, pp.125-126, ICCE-berlin 2013.

[2] Sung-Soo Park, Hwan Hur, Woon-Sung Lee, "A Study on the Biological Signal Changes due to Distraction during Operation in Vehicle Simulator Environment, Journal of the Ergonomics Society of Korea, Vol.29, No.1, pp..55-59, February 2010.
[3] Won-Hee Lee, Seong-Geon Bae, and Myung-Jin Bae, "A Study on Improving the Overloaded Speech Waveform to Distinguish Alcohol Intoxication using Spectral Compensation," IJET Vol.7, No.5, pp.235-237.

[4] Seong-Geon Bae, Myung-Jin Bae, "A New Speech Coding using Harmonics Emphasis Filter,” ISAAC 2013, AACL Vol. 1(2013), pp43-44.

[5] Ahn IK, Yun JS, Bae MJ, "A Study on the Effect of Automobile Engine Knocking Sound on Driver's Psychology", Proceedings of IEEK, pp.993-994, 2016.

[6] Won-Hee Lee, Myung-Sook Kim, and Myung-Jin Bae, Using valid-frame deviation to judgment of intoxication. Information: An International Interdisciplinary Journal, Vol. 18 (2015), 4131-4136.

[7] Won-Hee Lee, Seong-Geon Bae, and Myung-Jin Bae, "A Study on Improving the Overloaded Speech," International Journal of Applied Engineering Research ISSN 0973-4562 Volume 12, Number 23 (2017) pp. 14796-14801

[8] Seong-Geon Bae, Won-Hee Lee and Myung-Jin Bae, " A Study on Low Frequency Noise of Dehumidifier using Acoustic Charactristics," IJET Vol.8, No.1, pp.235-237.

[9] Won-Hee Lee, Myung-Jin Bae, "Reducing Errors of Judgment of Intoxication in Overloaded Speech Signal," IJET Vol.8, No.1(2016), pp.219-224.

[10] Seonggeon Bae, Myungsook Kim, and Myungjin Bae, "On Enhancement Signal Using Non-uniform Sampling in Clipped Signals for LTE Smart Phones ", IEEE ICCEberlin(2013), pp.125-126.

[11] Seonggeon Bae, Hyungwoo Park and Myungjin Bae, "On a New Enhancement of speech Signal using Nonuniform Sampling and Post Filter,'ICHIT 2012, LNCS 7425(2012), pp.723-729, Springer-Verlag.

[12] Zwicker, E., Fast, H. (1990): Psychoacoustics - Facts and Models, Springer - Verlag, Berlin. 